\title{
Clearing the detail coefficient of 2-D DWT Architecture based on Lifting scheme of 5/3 and 9/7 Filters
}

\author{
Dhafer Fakhery Hasan Al-layla \\ computer unit / Collage Medicine \\ University OF Mosul
}

\section{Received \\ $04 / 11$ / 2009}

Accepted

27 / 06 / 2010

الخلاصة

في هذا البحث تم تطبيق معمارية كفؤع ة لتنفيذ التحويل المويجي المقطع الثنائي الابعاد

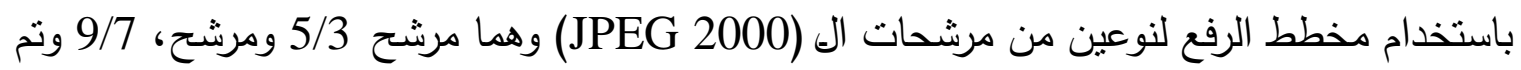

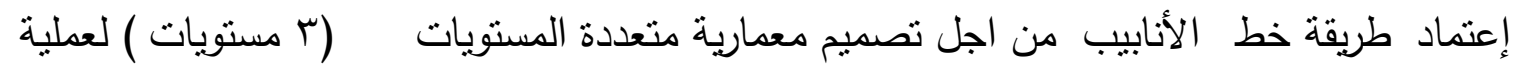

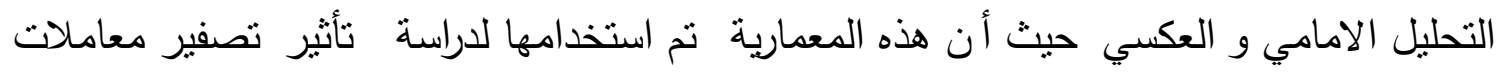
التقصيل (HH, HL, LH) على نوعية الصورة المسترجعة، وان معمارية التحويل المويجي المقطع الثائيً الابعاد المقترحة لمستوي واحد (one-level 2-D DWT) تنبى بالاعتماد على معمارية التحويل المويجي المقطع الاحادي الابعاد فق ط (1-D DWT) باستخدام (1-DDWT) كوحدة اسلسية. إن المعمارية المقترحة طورت لبناء نموذج التحويل المقطع الثنائي الابعاد لنّاث مستوييات (three-level 2-D DWT) وقياس نوعية الصورة باستخدام نسبة الاشارة الى الضوضاء (SNR)

\section{Abstract}

In This paper, An efficient architecture for the implementation of two dimensional discrete wavelet transform based on lifting scheme for two types of JPEG2000 filters (5/3 and 9/7 filter) are proposed. The pipelining method is applied in the proposed architecture to design multilevel (threelevel) architecture for both direction (forward and inverse) transform. After that, the proposed architecture is used to study the effect of clearing the detail coefficients HH, HL and LH sub-band images into the quality of 
reconstruction image, the proposed one-level two dimensional discrete wavelet transform architecture (one-level 2-D DWT) is built by using the one dimensional discrete wavelet transform (1-D DWT) only,then by using the 1-D DWT as basic element, the architecture are developed to built the three-level two dimensional discrete wavelet transform module (three-level 2-D DWT). The Matlab was used to simulate the proposed architectures and measure the quality of image by using the signal to Noise Ratio (SNR).

\section{Keyword: 2-D DWT, lifting scheme, 5/3 and 9/7 filter, 3-level 2-D DWT.}

\section{Introduction}

The discrete wavelet transform (DWT) is being increasingly used for image coding. This is due to the fact that DWT supports features like progressive image transmission (by quality, by resolution), ease of compressed image manipulation, Region of interest coding, etc. DWT has traditionally been implemented by convolution[1]. Such an implementation demands both a large number of computations and a large storage features that are not desirable for either high-speed or low-power applications. Recently, a lifting-based scheme that often requires far fewer computations has been proposed for the DWT [2], [3]. The main feature of the lifting based DWT scheme is to break up the high pass and low pass filters into a sequence of upper and lower triangular matrices and convert the filter implementation into banded matrix multiplications [2], [3]. Such a scheme has several advantages, including "in-place" computation of the DWT, integer-to-integer wavelet transform (IWT), symmetric forward and inverse transform, etc. Therefore, it comes as no surprise that lifting has been chosen in the upcoming JPEG2000 standard. In the JPEG2000 verification model (VM) Version 8.5, the following wavelet filters have been proposed: $(5,3)$ (the high pass filter has five taps and the low pass filter has three taps), ( 9 , $7), \mathrm{C}(13,7), \mathrm{S}(13,7),(2,6),(2,10)$, and $(6,10)$. To be JPEG2000 compliant, the coder should be able to at least provide a $(5,3)$ filter in lossless mode and a $(9,7)$ filter in lossy mode. In this Paper, an unified architecture was proposed which is capable of executing all the filters mentioned above using the lifting scheme. Since novel shift-Accumulator (SA-ALU) centric lifting scheme architecture is proposed to reduce the hardware utilization and the time delay of the operation [4]. The architectures are mostly folded and can be broadly classified into serial architectures (where the inputs are supplied to the filters in a serial manner) and parallel architectures (where the inputs are supplied to the filters in a parallel manner [5]). The serial architectures are either based on systolic arrays that interleave the computation of outputs of different levels to reduce 
storage and latency [6] or on digit pipelining, which implements the filter bank structure efficiently [7]. A scalable architecture in [8] was proposed to implement as VLSI architecture for 2D DWT with $100 \%$ hardware utilization based on lattice structure.

This paper is organized as follows. Section 2 describes the convolution and lifting scheme of $5 / 3$ and $9 / 7$ filters of discrete wavelet transform. In section 3 , the analysis of 1-D forward and inverse discrete wavelet transform module for both $5 / 3$ and 9/7 filter are explained. The one and multi-level two dimensional forward discrete wavelet transform module are listed in section 4 , and the inverse module for one and multilevel are shown in section 5 . The clearing the detail coefficients are explained in section 6. Finally the conclusions are declared in section 7.

\section{DISCRETE WAVELET TRANSFORM}

\section{1 convolution method}

The traditional forward and inverse1-D DWT can be realized by convolution based implementation [9], [10]. In the forward transform, the input sequences $\mathrm{x}[\mathrm{n}]$ are down sampling and filtered by the low-pass filters ha[n] and high-pass filters ga[n] as shown in Fig.1 [9].

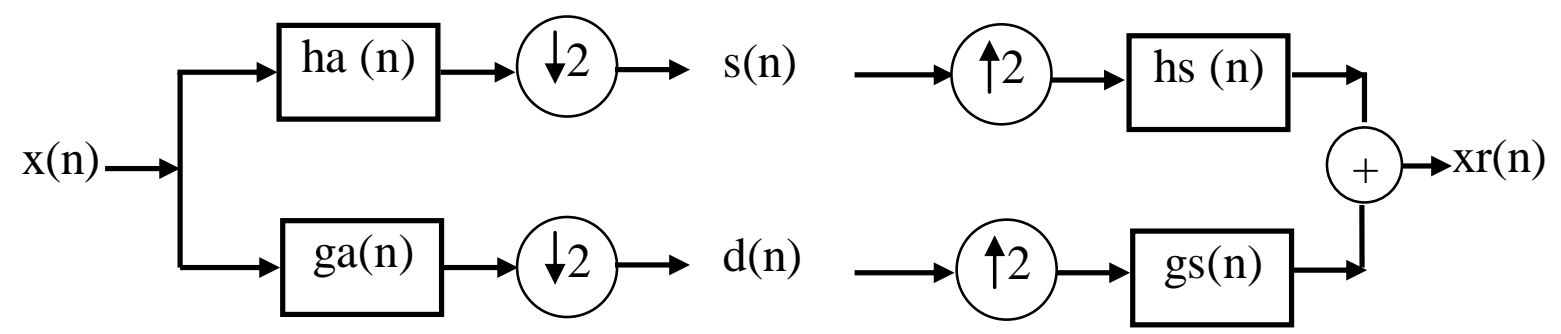

Fig.1 the convolution method to represent the discrete wavelet transforms.

To obtain the low-pass and high-pass DWT coefficients, $s[n]$ and $d[n]$. The equations may be written as follows:

$$
\begin{aligned}
& s(n)=\sum_{k=0}^{M-1} x(k) h a(2 n-k) \\
& d(n)=\sum_{k=0}^{M-1} x(k) \operatorname{g} a(2 n-k)
\end{aligned}
$$

Where

$\mathrm{n}$ : the index of signal which is represent as matrix.

$\mathrm{x}(\mathrm{n})$ : the input data.

ha(n): the low pass filter of the analysis level.

$\mathrm{ga}(\mathrm{n})$ : the high pass filter of the analysis level.

$\mathrm{s}(\mathrm{n})$ : the approximation coefficient. 
$\mathrm{d}(\mathrm{n})$ : the detail coefficient.

For the inverse transform, the DWT coefficients are up sampling and filtered by another set of low-pass $h \mathrm{~s}[n]$ and high-pass $g \mathrm{~s}[n]$ filters to reconstruct the original data $\mathrm{xs}[n])$, which is described as follows:

$$
x s=\sum_{k=0}^{M-1} s(k) h s(n-k)+d(k) g s(n-k)
$$

\section{2 lifting scheme}

The lifting-based forward 1-D discrete wavelet transform was derived from Daubenchies and Sweldens [1] [2]. As shown in Fig.1, it indicates that the traditional filter banks wavelet transform could be decomposed into several lifting steps. As the lifting-based DWT also reduces the hardware complexity of the traditional filter bank Implementation [11]. The lifting scheme consists of the following three steps to decompose the samples, as shown in Fig.2.

(1) Split step: The input samples 1 is split into even samples and odd samples;

(2) Predict step(P): The even samples are multiplied by the predict factor and then the results are added to the odd samples to generate the detailed coefficients;

(3) Update step(U): The detailed coefficients computed by the predict step are multiplied by the update factors and then the results are added to the even samples to get the coarse coefficients.

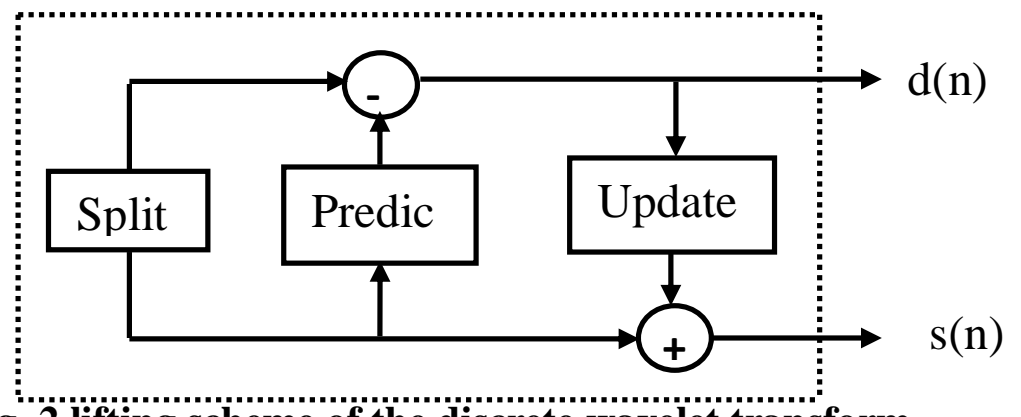

Fig. 2 lifting scheme of the discrete wavelet transform

\subsection{1 lifting scheme in $5 / 3$ filter}

The filter coefficient of low pass filter $h_{a}[n]=[-1 / 8,1 / 4,3 / 4,1 / 4,-1 / 8]$.

The filter coefficient of high pass filter $\mathrm{g}_{\mathrm{a}}[\mathrm{n}]=[-1 / 2,1,-1 / 2]$, [12].

So the three steps of lifting scheme can be written as the following equations:

1. Splitting Step:

$d_{i}^{0}=x_{2 i+1}(4)$

$s_{i}^{\mathrm{O}}=\boldsymbol{x}_{2 i}(5)$

2. Predict step: 


$$
d_{i}^{1}=d_{i}^{\mathrm{O}}+\alpha\left(s_{i}^{\mathrm{O}}+s_{i+1}^{\mathrm{O}}\right)
$$

3. Update Step:

$$
s_{i}^{1}=s_{i}^{0}+\beta\left(d_{i-1}^{1}+d_{i}^{1}\right)
$$

Where

$\alpha$ : the $5 / 3$ filter coefficient in predict step.

$\beta$ : the $5 / 3$ filter coefficient in update step.

$\alpha=-0.5, \beta=0.25$ [12].

The lifting scheme of 5/3 filter can be determined as shown in Fig. 3 [13].

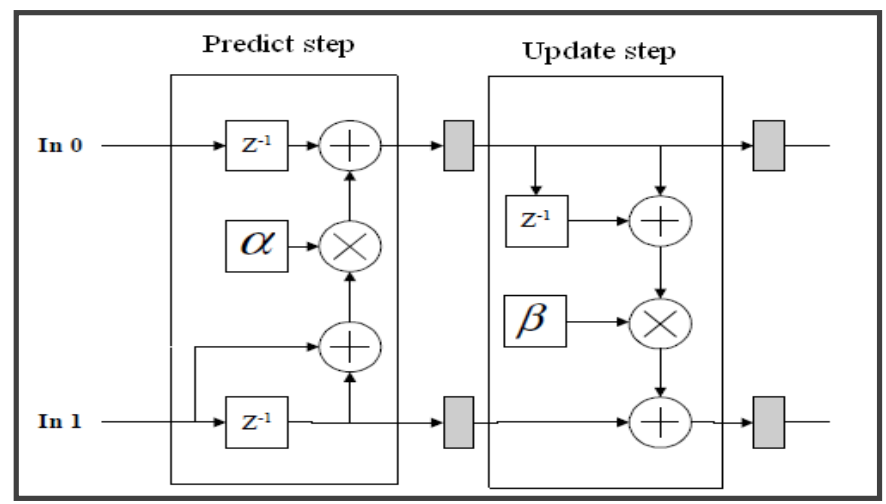

Fig.3 the lifting scheme of $5 / 3$ filter.

\subsubsection{The lifting scheme in $9 / 7$ filter}

The lifting scheme of 9/7 filter [12] as shown on fig. 4 consist of

1. Splitting Step:

$$
\begin{aligned}
& d_{i}^{0}=x_{2 i+1}(8) \\
& \boldsymbol{s}_{\boldsymbol{i}}^{\mathbf{O}}=\boldsymbol{x}_{2 i}
\end{aligned}
$$

2. Lifting Step:

(First Lifting Step)

$d_{i}^{1}=d_{i}^{\mathrm{O}}+\alpha\left(s_{i}^{\mathrm{O}}+s_{i+1}^{\mathrm{O}}\right)$ (Predictor) (10)

$s_{i}^{1}=s_{i}^{0}+\beta\left(d_{i-1}^{1}+d_{i}^{1}\right)$ (Updater) (11)

(Second Lifting Step)

$$
\begin{array}{ll}
d_{i}^{2}=d_{i}^{1}+\gamma\left(s_{i}^{1}+s_{i+1}^{1}\right) & \text { (Predictor) (12) } \\
s_{i}^{2}=s_{i}^{1}+\delta\left(d_{i-1}^{2}+d_{i}^{2}\right) & \text { (Updater) (13) }
\end{array}
$$

\section{Where}

$\alpha$ : the $9 / 7$ filter coefficient in the first lifting predict step. $\beta$ : the $9 / 7$ filter coefficient in the first lifting update step. $\gamma$ : the $9 / 7$ filter coefficient in the second lifting predict step. $\delta$ : the $9 / 7$ filter coefficient in the second lifting update step. $\alpha=-1.5, \beta=-0.0625, \gamma=0.7998046875, \delta=0.46875$ 
Clearing the detail coefficient of 2-D DWT Architecture based on Lifting scheme ...

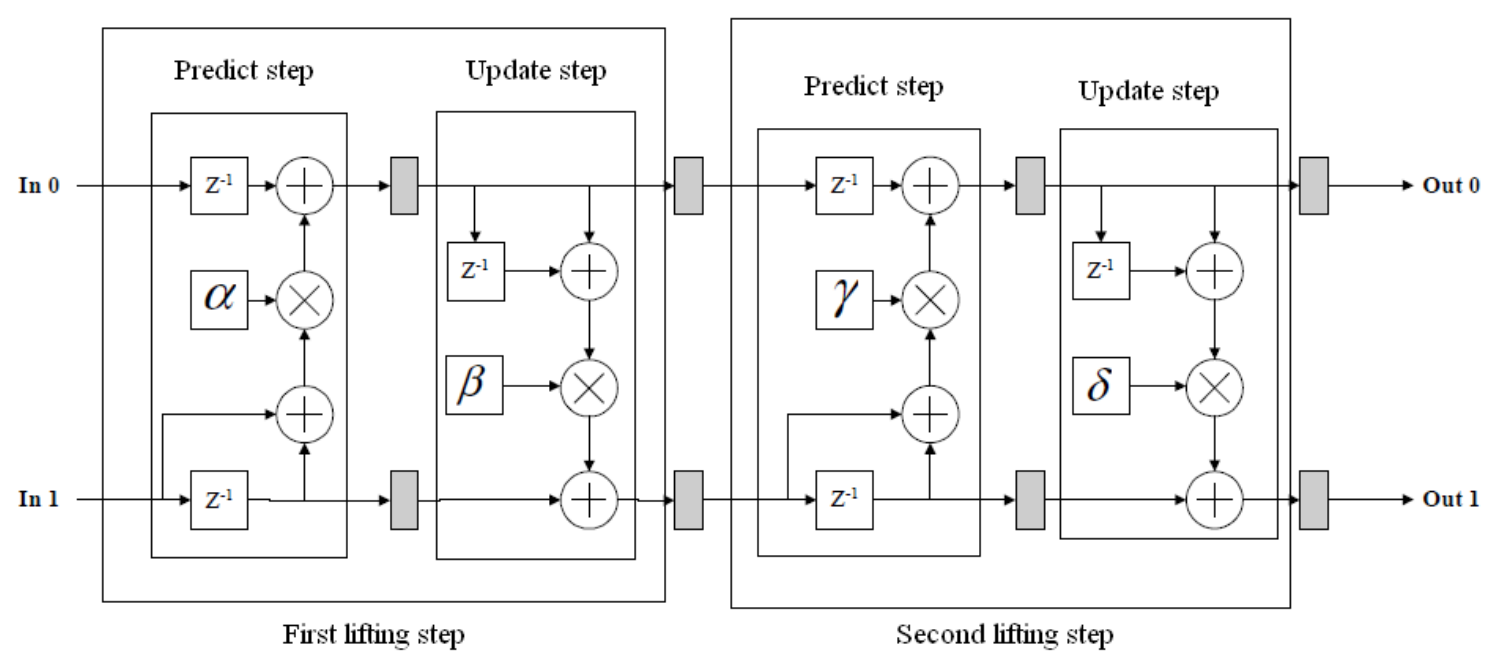

Fig. 4 The lifting scheme of 9/7 filter.

\section{One Dimensional Discrete Wavelet Transform module (1-D DWT ):}

To design the forward and inverse 1-D DWT module, the type of filter that is used should be determined. In this paper, two type of JPEG2000 filter are used (5/3 and 9/7 filter), so the first module are designed depends on $5 / 3$ filter and the second module are designed depends on 9/7 filter [14].

\subsection{The 1-D Forward DWT module (1-D FDWT) of 5/3 and 9/7 filter:}

The forward 1-D DWT of 5/3 filter are designed based on lifting scheme as shown in Fig.5.

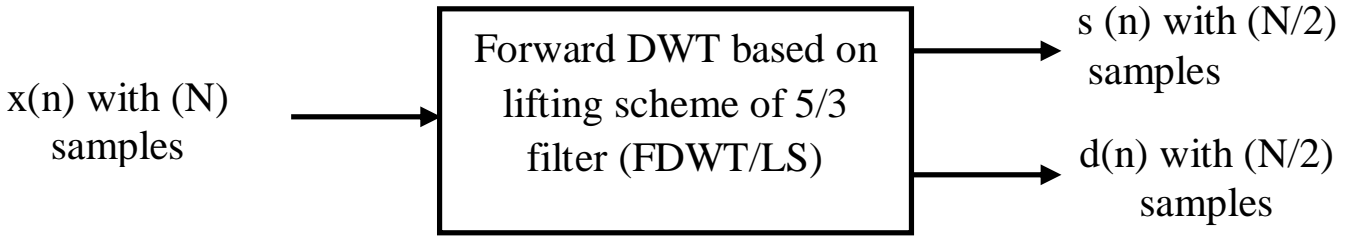

Fig. 5 The forward lifting scheme block of 1-D DWT based on 5/3 filter.

And the module of forward 1-D DWT of 9/7 filter are built as shown in fig.6 depends on equations (8)-(13).

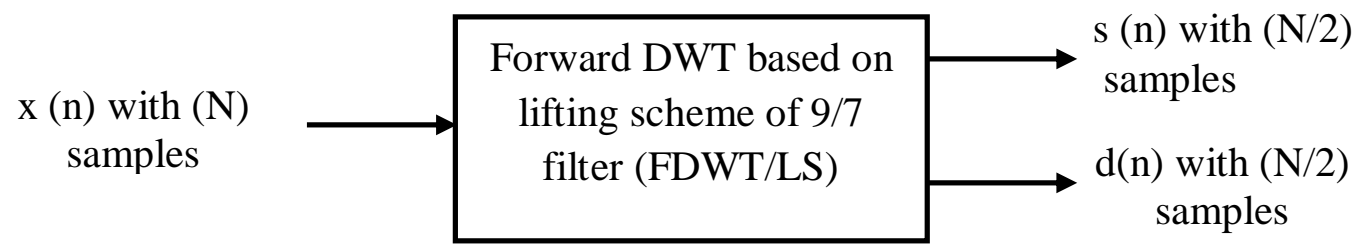

Fig. 6 The forward lifting scheme block of 1-D DWT based on 9/7 filter. 
The input signal $\mathrm{x}(\mathrm{n})$ is one dimension signal,but in two dimensional signal like image which has a size equal to $\left(\mathrm{N}^{*} \mathrm{~N}\right)$, the above modules must be developed to suit the 2-dimensional input.

\subsection{The 1-D Inverse DWT module (1-D IDWT) of 5/3 and 9/7 filter:}

The inverse module of $5 / 3$ filter can be designed depends on the symmetrical computation of lifting scheme as shown in Fig. 7.

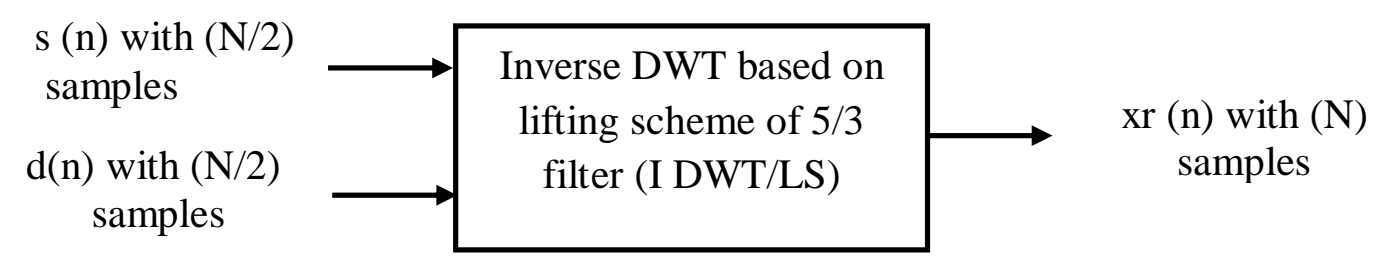

Fig. 7 The inverse DWT module based on lifting scheme of 5/3 filter.

The inverse module of 9/7 fitter is designed as shown in Fig. 8.

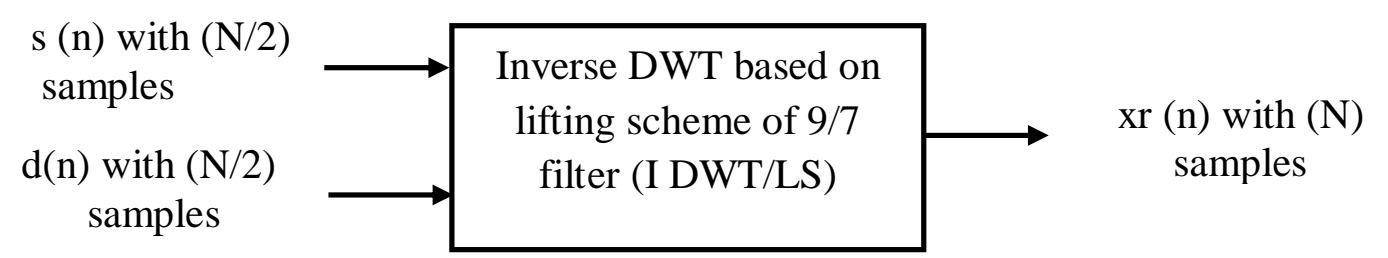

Fig. 8 The inverse DWT module based on lifting scheme of $5 / 3$ filter.

\section{One-level two Dimensional Forward Discrete Wavelet} Transform (one-level 2-D FDWT) module

The 2-D FDWT are build depends on the ROW-COLUMN fashion on a block of data size $\mathrm{N}^{*} \mathrm{~N}$, i.e. the pixels from the first row of the input image are read from the 1-D DWT Block1 as shown in Fig.9(a) and calculate the approximation and detail coefficients, in 2-D DWT the approximation coefficient represent the low frequency data (L) and the detail coefficients is representing the high frequency data $(\mathrm{H})$ which are stores into intermediate memory bank $(\mathrm{N} * \mathrm{~N})$, this calculation continuous until the last pixel of the last row. At this point all the operation is done at every row of the image, so these operations are called row discrete wavelet transform. After that the 1-D DWT Block2 begins to calculate the 1-D DWT in every column of approximation coefficients, at the same time the 1-D DWT Block3 reads the detail coefficients from the intermediate memory bank and calculate the DWT in each column. This kind of operation is called the column discrete wavelet transform. The 1-D DWT Block 2 generate the approximation and detail coefficients from the low frequency data so, the approximation coefficients from 1-D DWT Bolck2 is representing the low frequency data which generated from the low frequency data (LL sub-band image) and the detail coefficients from 1-D DWT Block2 is representing the high frequency data which generated from the low frequency data (LH sub-band image). The 1-D DWT Block3 is calculating the approximation (low frequency) and detail (high frequency) coefficients from high frequency data $\mathrm{HL}$ and $\mathrm{HH}$ sub-band images respectively. The one-level 2-D FDWT consist of 3 
processor elements and intermediate memory bank, the internal design of the processor elements are similar but the internal design depends on the type of the used filter either $5 / 3$ or $9 / 7$ filters. The size of each sub-band images (LL, $\mathrm{LH}, \mathrm{HL}$ and $\mathrm{HH}$ ) is $\mathrm{N}^{2} / 4$. The LL sub-band image is represent the approximation data of image which includes the must important information of the image and another three sub-band images ( $\mathrm{LH}, \mathrm{HL}$ and $\mathrm{HH}$ ) is the detail coefficients of the image.

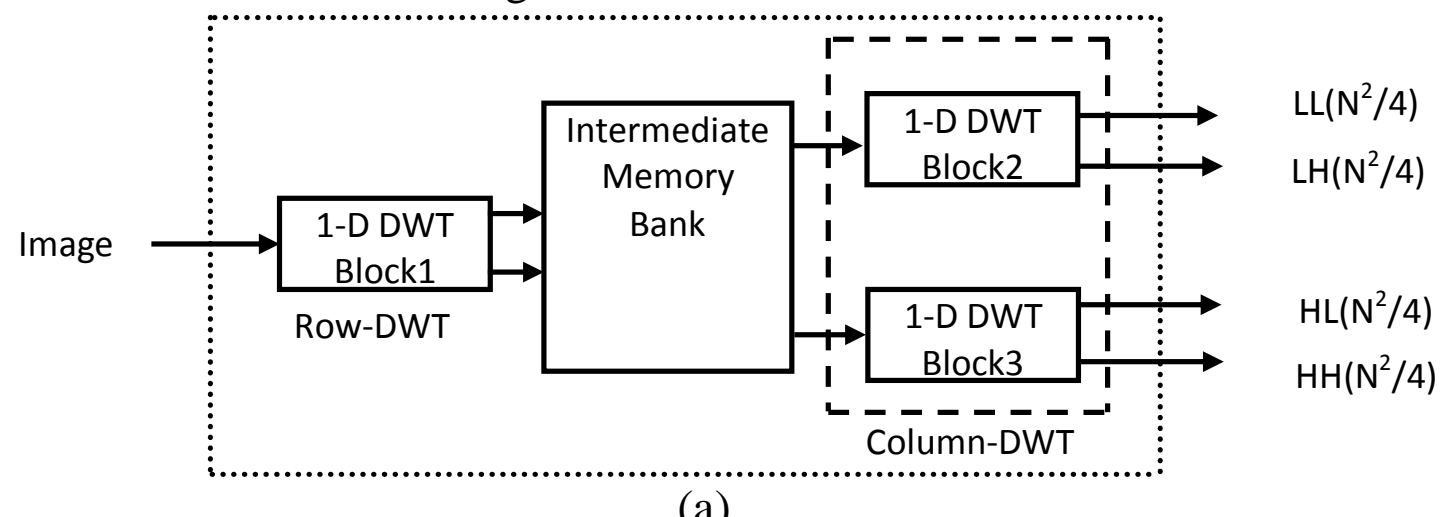

(a)

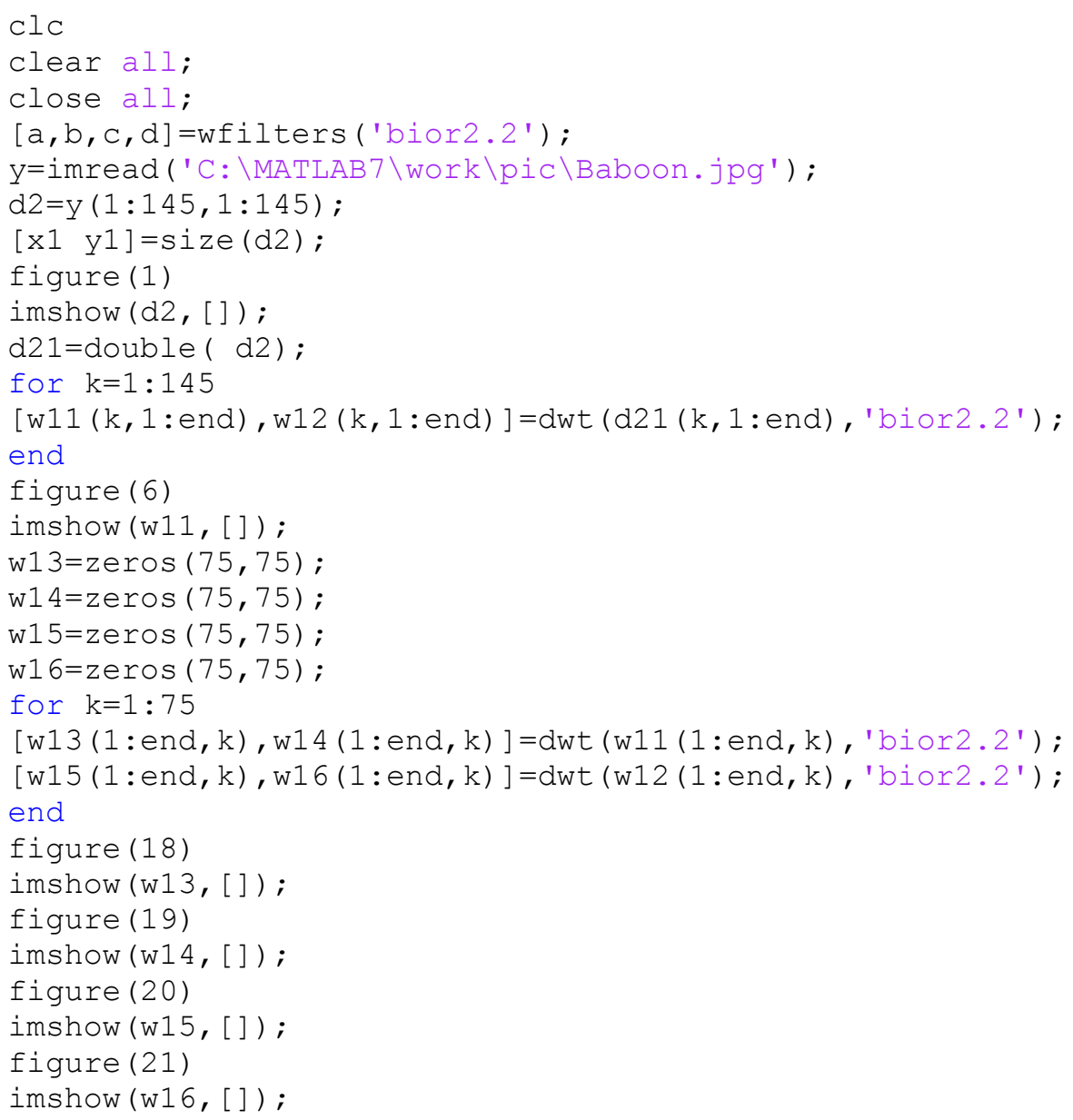

(b)

Fig.9 (a) One-level 2-D FDWT module, (b) the Matlab code of 2-D FDWT. 


\section{1 the Multi-level Forward 2-D DWT module:}

The multi-level of 2-D DWT can be designed depends on one-level 2-D FDWT module as shown in Fig. 10. At the beginning, the Multiplier (MUX) entered the data from the image to calculate the first level of 2-D FDWT and all LL sub-band image are stored into memory buffer $\left(\mathrm{N}^{2} / 4\right)$ which the size of input image is $\mathrm{N}^{*} \mathrm{~N}$ and the other sub-band images are stored into the external memory. After the first level of 2-D FDWT is finished, the MUX begins to read the data from memory buffer $\left(\mathrm{N}^{2} / 4\right)$ and the second level of 2D DWT is start and all the operation are repeated until the data from memory buffer $\left(\mathrm{N}^{2} / 4\right)$ is finished, at the same time the LL sub-band image of the second level is stored into memory buffer, here the used Size of the memory buffer is $\mathrm{N}^{2} / 16$. The other sub-band images of the second level (LH, $\mathrm{HL}$ and $\mathrm{HH}$ ) are stored into external memory. In this paper, the architecture was build to calculate the three-level 2-D FDWT based on lifting scheme as shown in Fig.11.

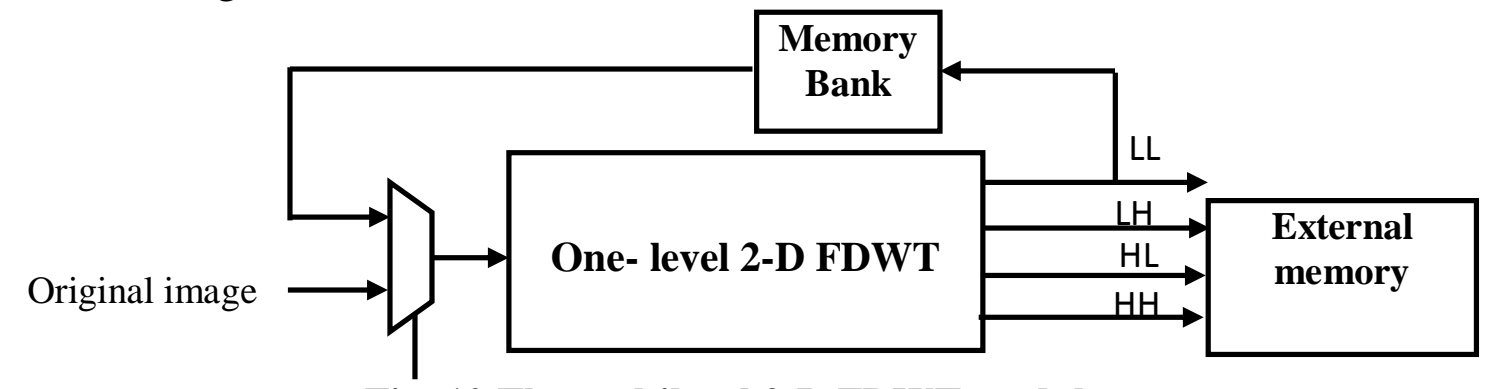

Fig. 10 The multilevel 2-D FDWT module.
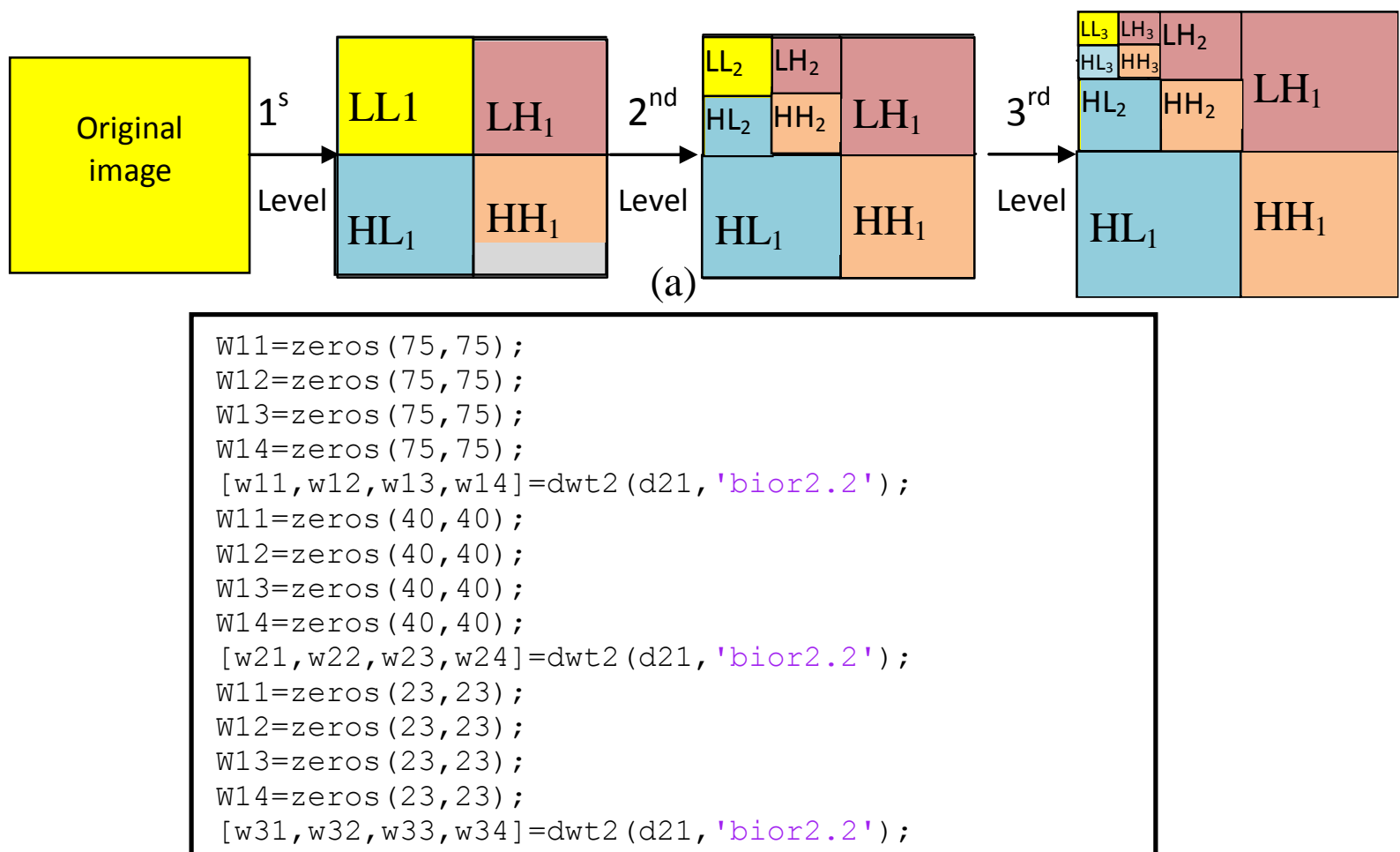

(b)

Fig. 11 (a) Three-levels Forward 2-D DWT module, (b) The Matlab code of threelevel 2-D FDWT. 


\section{One-level two Dimensional Inverse Discrete Wavelet Transform(one-level 2-D IDWT) module:}

The inverse 2-D DWT as shown in Fig.12, the 1-D IDWT Block1 read the LL, LH sub-band images and the 1-D IDWT Block2 read the HL, HH sub-band images in parallel manner to calculate the reconstruction low and high frequency data which are stored into internal memory bank, after the 2 modules is finished their operation, the 1-D IDWT Block3 begins to read the reconstruction data from the internal memory bank and generate the reconstruction image $\mathrm{X}_{\mathrm{r}}\left(\mathrm{N}^{*} \mathrm{~N}\right)$.

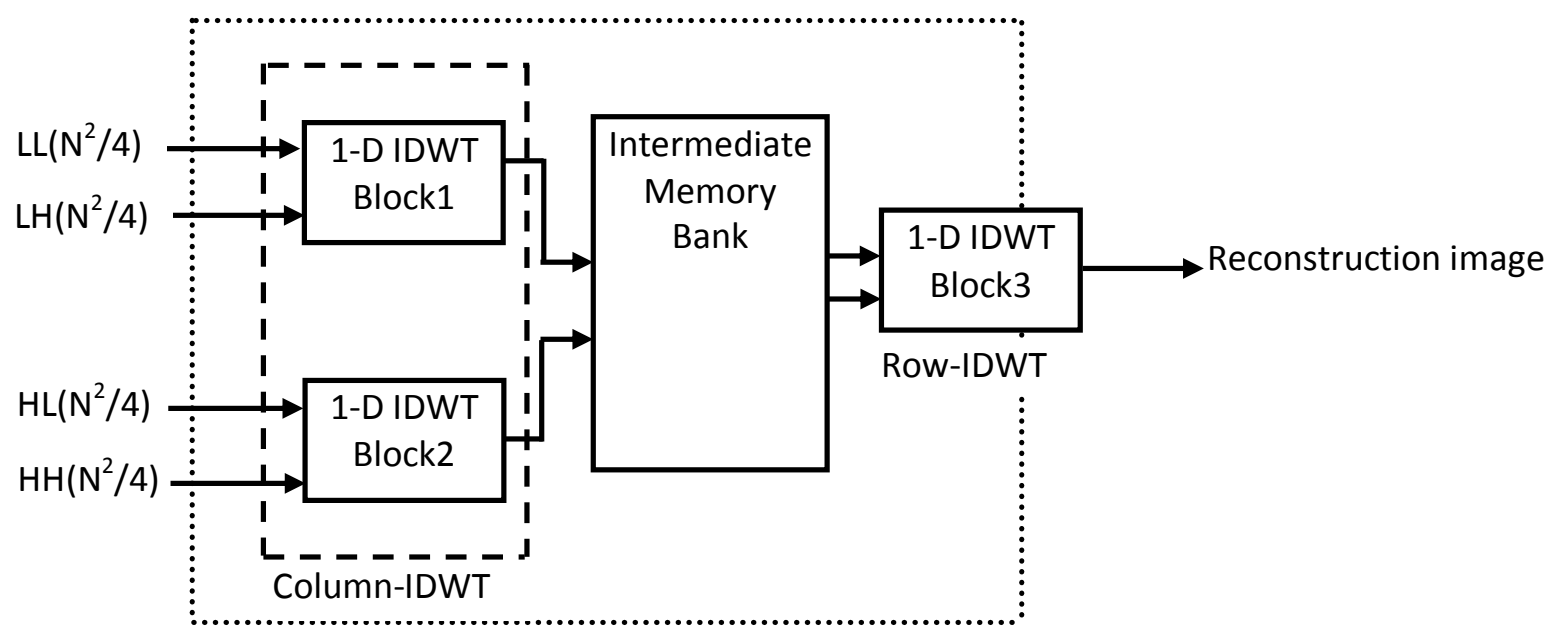

(a)

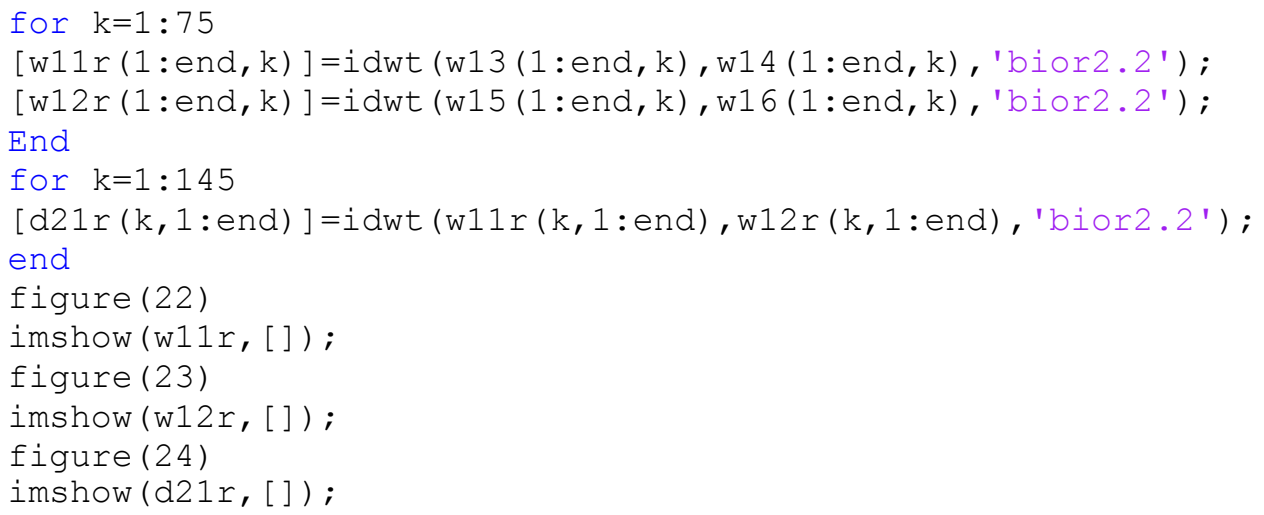

(b)

Fig.12 (a) One-level two Dimensional Inverse Discrete Wavelet Transform, (b) The Matlab code of 2-D IDWT.

\subsection{The Multi-level Inverse 2-D DWT module:}

The inverse multilevel module can be designed depends on one-level module as shown in Fig.13. The module begins to read the LL, LH, HL and 


\section{Dhafer Fakhery Hasan Al-layla}

HH sub-band images of the third level 2-D FDWT which are stored into external memory and calculate the inverse 2-D DWT to reconstruct the LL sub-band image of the second level ( $\left.\mathrm{LLr}_{2}\right)$ which is stored into memory bank $\left(\mathrm{N}^{2} / 4\right)$ as shown in Fig.13. After the finishing of reconstructing the data of the second level, the module begins to read the $\mathrm{LLr}_{2}$ and the $\mathrm{LH}, \mathrm{HL}$ and $\mathrm{HH}$ sub-band images of the second level of 2-D FDWT of the external memory as shown in Fig.14.

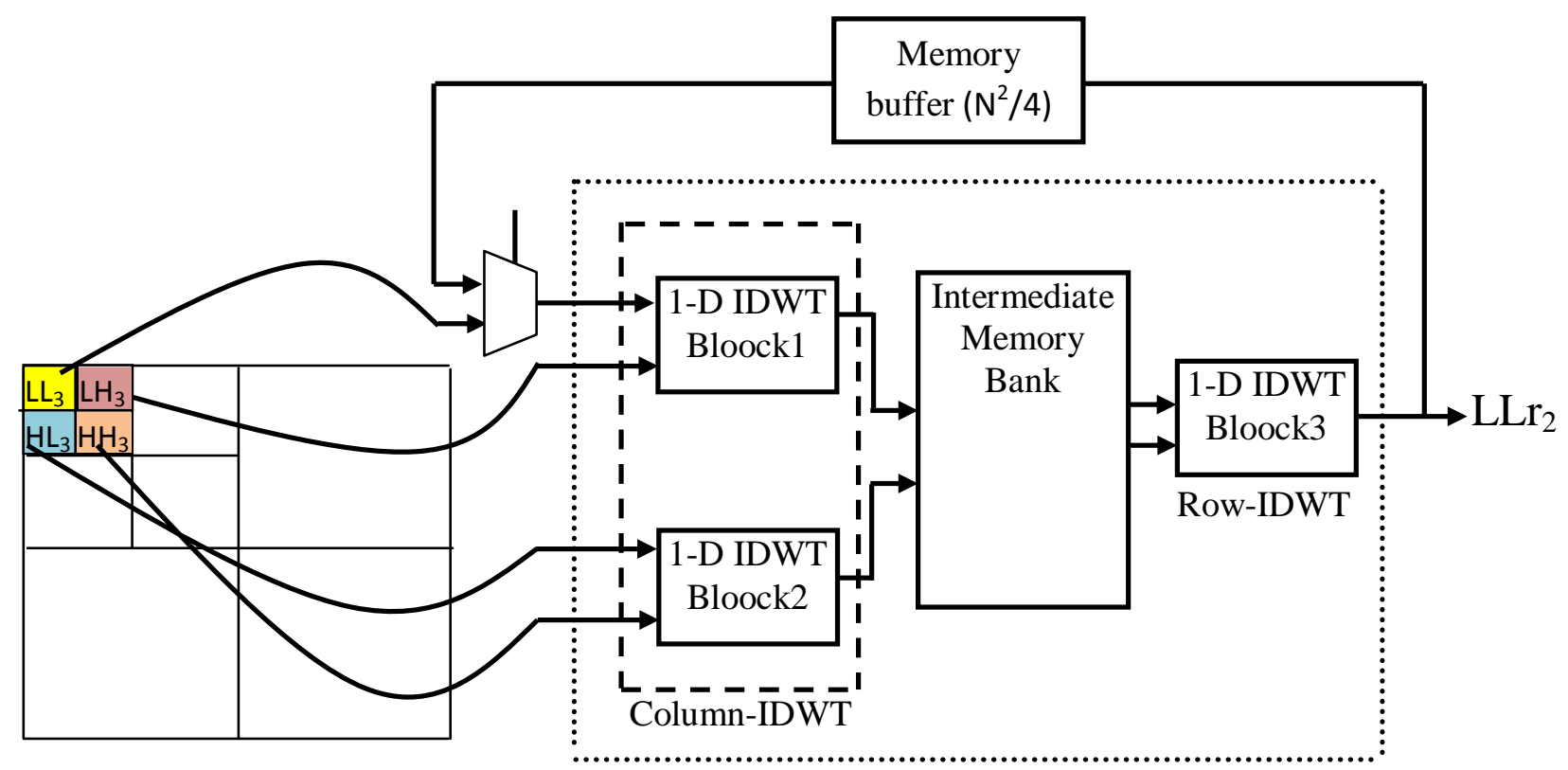

Fig.13 The implementation of first level inverse 2-D IDWT.

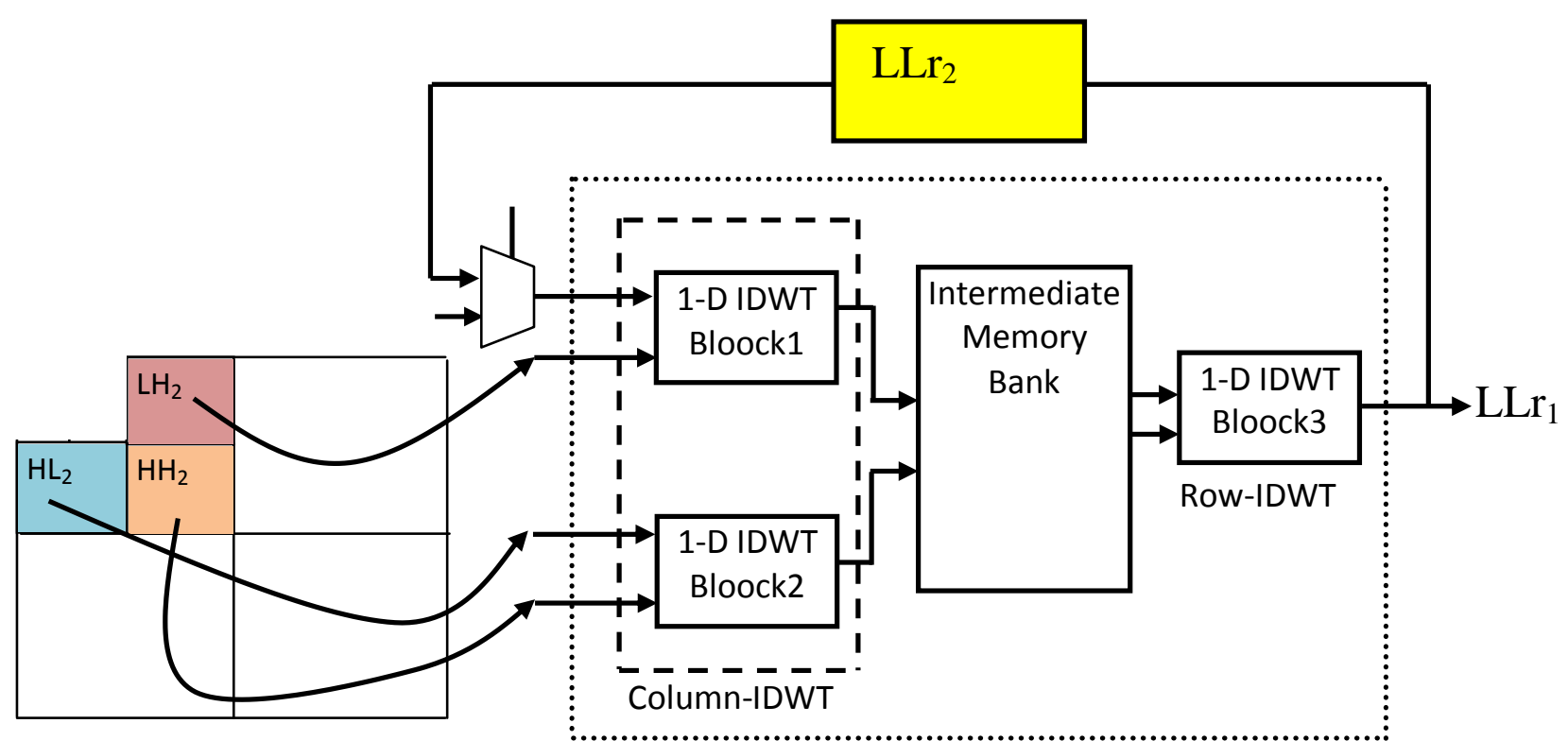

Fig.14 The implementation of second level inverse 2 -D IDWT. 
And generate the reconstruction data $\left(\mathrm{LLr}_{1}\right)$ and is stored into memory bank $\left(\mathrm{N}^{2} / 4\right)$ Finally the module read the $\mathrm{LLr}_{1}$ from memory bank $\left(\mathrm{N}^{2} / 4\right)$ and the LH,HL,HH of first level of 2-D FDWT from external memory and generate the reconstruction image (Xr) as shown in Fig.15.

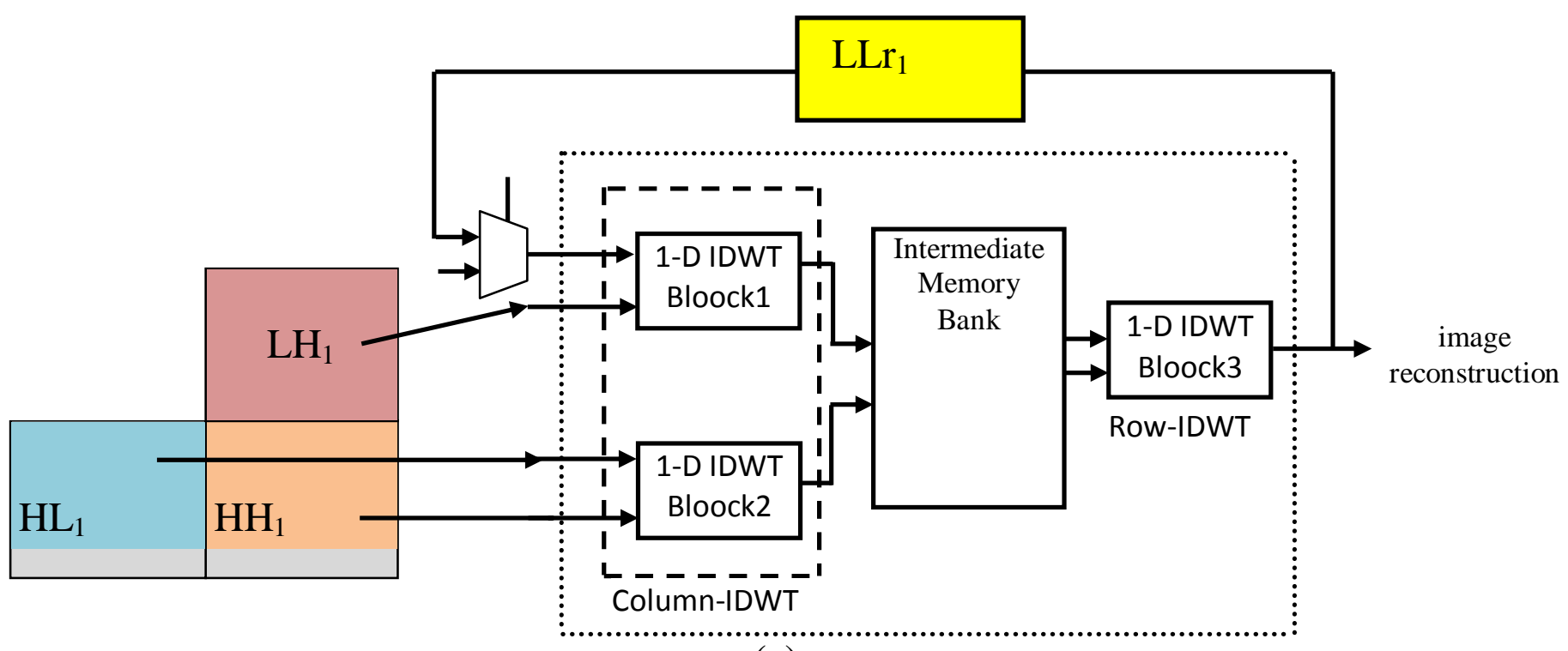

(a)

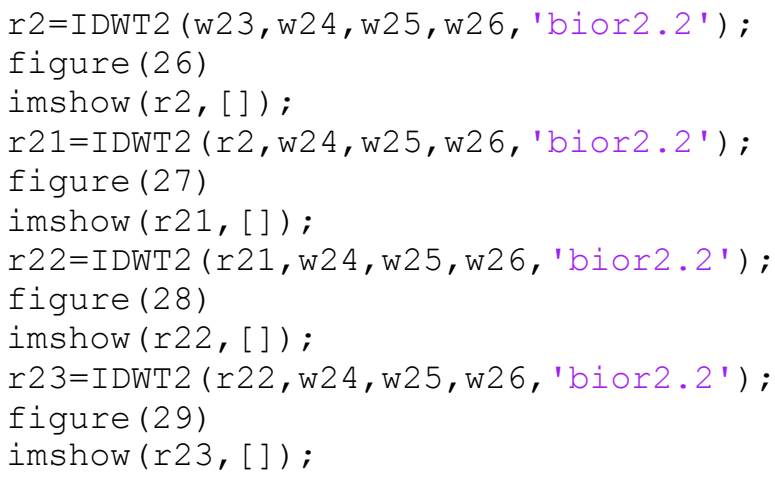

(b)

Fig.15 (a) The implementation of third level inverse 2-D IDWT, (b) The Matlab code of third level 2-D IDWT.

\section{Clearing the Detail Coefficients}

6.1 Clearing the HH sub-band image

After designing the forward and inverse 2-D DWT architectures for both 5/3 and 9/7 filter, the smoothing processing is beginning by clearing the $\mathrm{HH}$ sub-Band image which is produces by the one-level forward 2-D DWT module as shown in Fig. 16. 


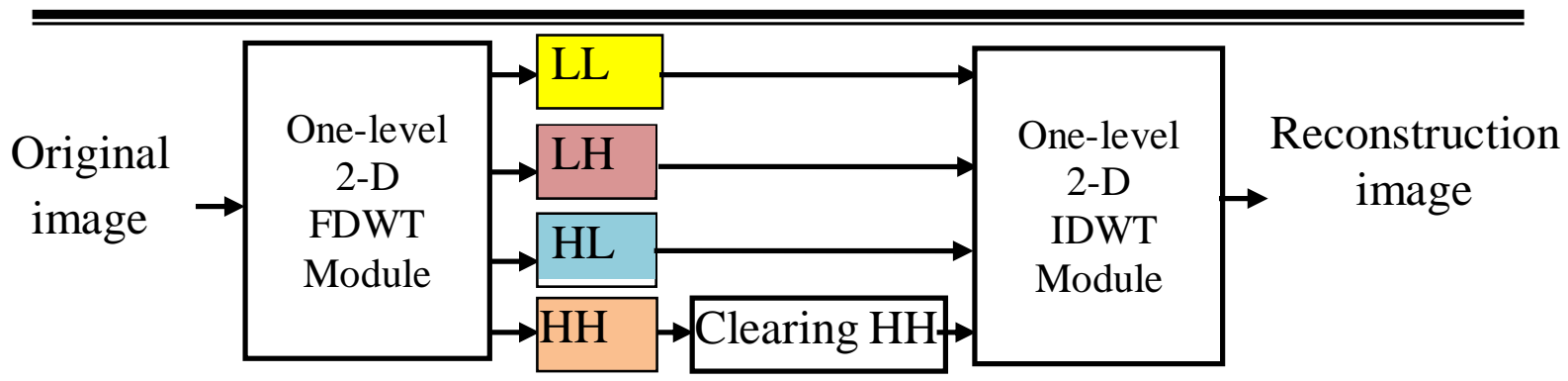

Fig.16 The implementation of clearing process in the HH sub-band image.

Then reconstruct the image by using the one-level inverse 2-D DWT module, The quality of reconstructed image can be measured by using the Signal to Noise ratio (SNR) criteria as the following equation:

$$
S N R=10 \log \sum_{i=0}^{N-1} \sum_{j=0}^{M-1} \frac{x(i, j)^{2}}{(x(i, j)-x r(i, j))^{2}}
$$

This type of clearing $\mathrm{HH}$ sub-Band image is called smoothing image because this processing erase the high frequencies from the image and smooth the edge of image, thus, one of the most popular type of enhancement image is clearing $\mathrm{HH}$ sub-band image. This clearing is reducing the noise from the noisy image because the noise is found in the high frequency range, so when deleting this range by clearing $\mathrm{HH}$ sub-band image, the noise is deleted too. The values of SNR that are calculated by using Matlab to simulate the one-level forward and inverse 2-D DWT module is listed by Table.1. The reconstruction images of 5/3 and 9/7 filtersas shown in Fig. 17.

Table.1 The SNR results from clearing HH sub-band image.

\begin{tabular}{|c|c|c|}
\hline $\begin{array}{c}\text { Images } \\
\text { 145X145 }\end{array}$ & $\begin{array}{c}\text { SNR(dB) by } \\
\text { using 5/3 filter }\end{array}$ & $\begin{array}{c}\text { SNR(dB) by } \\
\text { using 9/7 filter }\end{array}$ \\
\hline Barbara & 63.25 & 63.58 \\
\hline Baboon & 66.53 & 68.35 \\
\hline papers & 77.55 & 80.79 \\
\hline Camera man & 52.94 & 54.96 \\
\hline
\end{tabular}

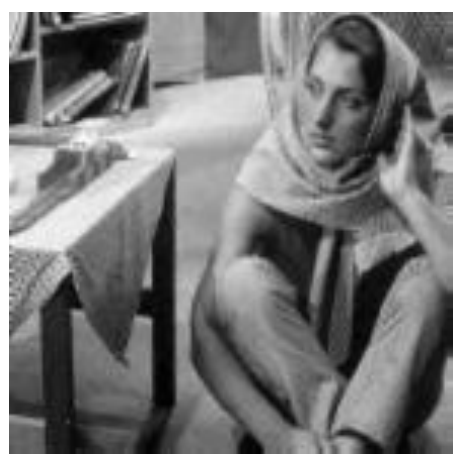

Reconstructed Image of

Barbara after clearing $\mathrm{HH}$ in 5/3 Filter

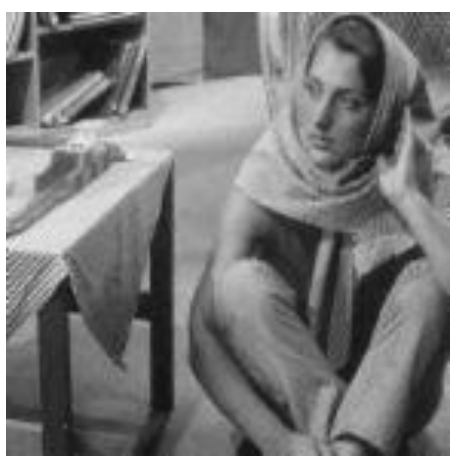

The original Image of Barbara

\section{Reconstructed Image of}

Barbara after clearing $\mathrm{HH}$ in 9/7 Filter 


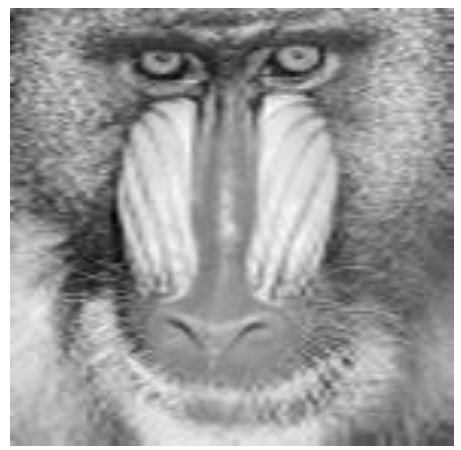

Reconstructed Image of Baboon after clearing $\mathrm{HH}$ in 5/3 Filter

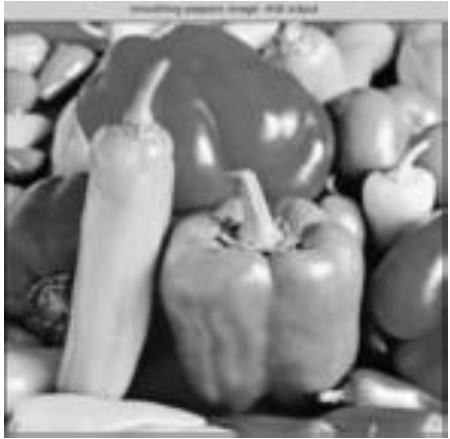

Reconstructed Image of Paper after clearing $\mathbf{H H}$ in $\mathbf{5 / 3}$ Filter

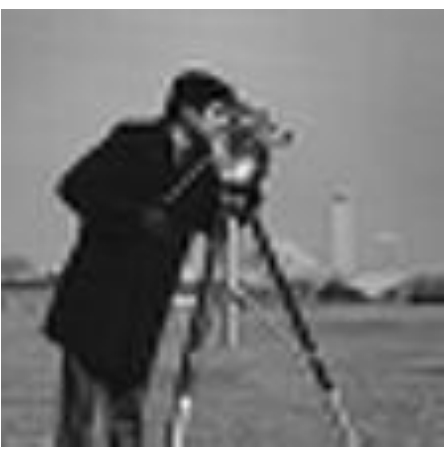

Reconstructed Image of Camera man after clearing $\mathrm{HH}$ in 5/3 Filter

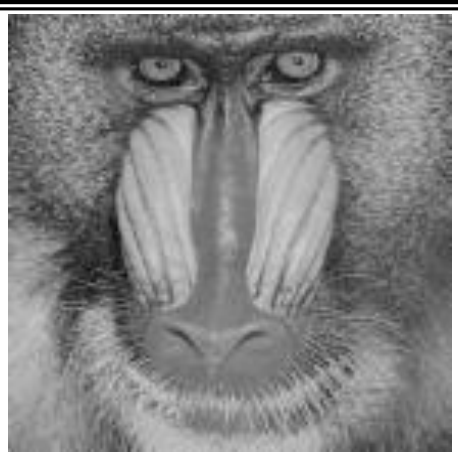

The original Image of Baboon

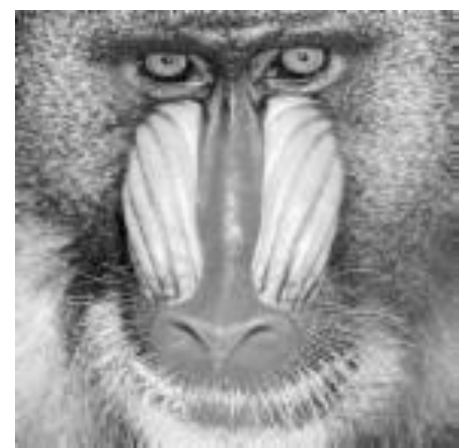

Reconstructed Image of Baboon after clearing $\mathrm{HH}$ in 9/7 Filter

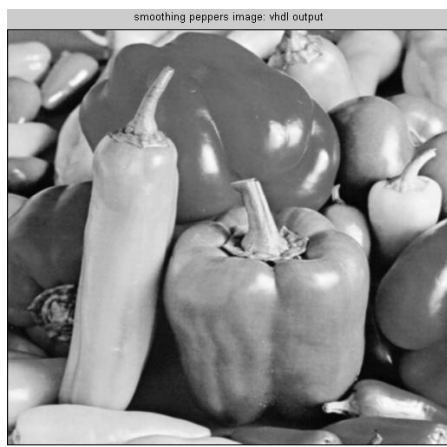

The original Image of Paper

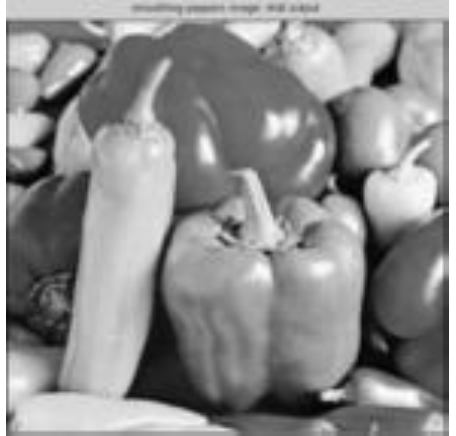

Reconstructed Image of Paper after clearing $\mathrm{HH}$ in 9/7 Filter

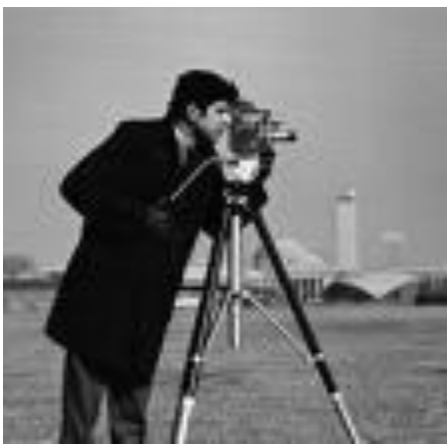

The original Image of Camera man

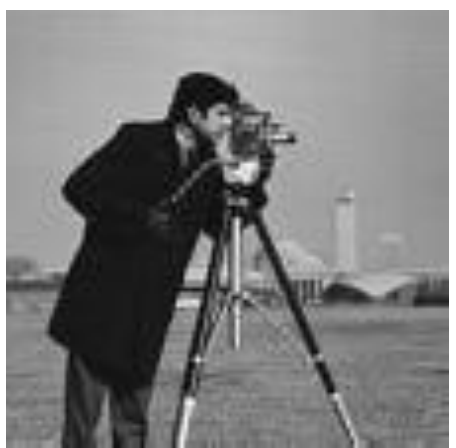

Reconstructed Image of Camera man after clearing $\mathrm{HH}$ in 9/7 Filter

Fig. 17 The reconstruction images of 5/3 and 9/7 filters. 
In order to clear the high frequencies from every row of the image, it must be clear the HL and HH sub-band image as shown in Fig.18. The SNR for this architecture are listed by Table.2. The reconstruction images of $5 / 3$ and 9/7 filters as shown in Fig. 19.

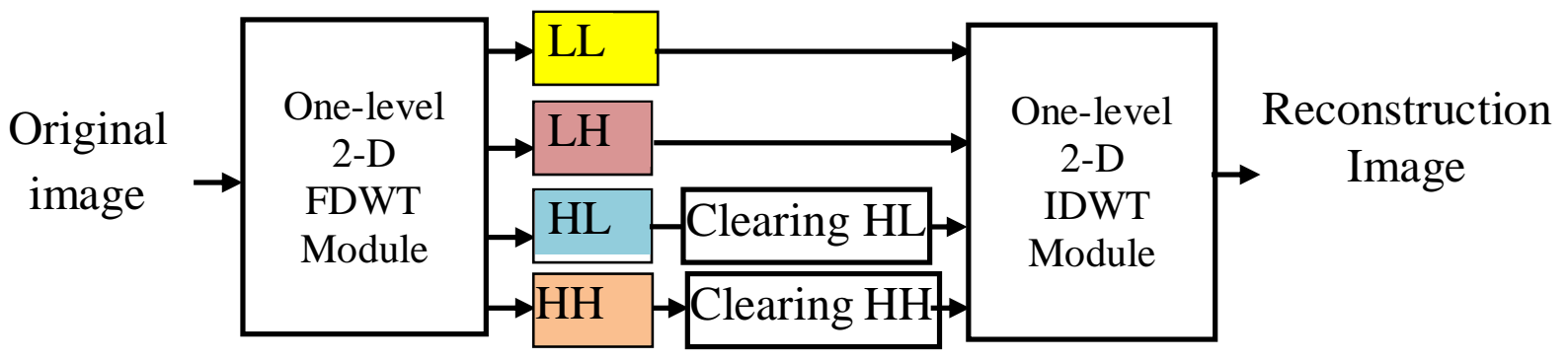

Fig.18 The implementation of clearing process in the HH and HL sub-band image.

\subsection{Clearing The HH, HL and LH sub-band images}

In order to clear the high frequencies from each column and each row of the image, it should be clear the HH, HL and LH sub-band images and all the result illustrated in Table.3. The reconstruction images of 5/3 and 9/7 filters as shown in Fig 20.

Table.2 The SNR results from clearing HH and HL sub-band images.

\begin{tabular}{|c|c|c|}
\hline $\begin{array}{c}\text { Images } \\
\text { 145X145 }\end{array}$ & $\begin{array}{c}\text { SNR(dB) by } \\
\text { using 5/3 filter }\end{array}$ & $\begin{array}{c}\text { SNR(dB) by } \\
\text { using 9/7 filter }\end{array}$ \\
\hline Barbara & 49.51 & 52.68 \\
\hline Baboon & 54.54 & 56.67 \\
\hline papers & 33.87 & 33.21 \\
\hline Camera man & 35.04 & 36.68 \\
\hline
\end{tabular}

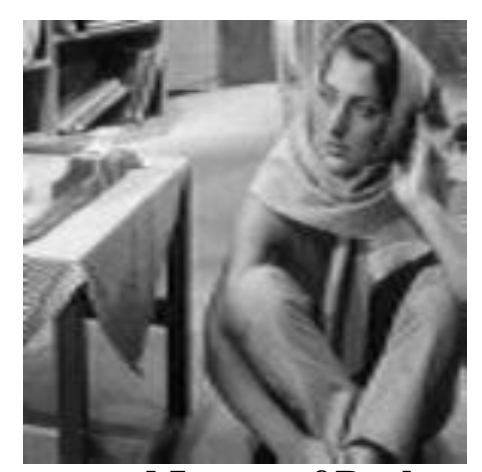

Reconstructed Image of Barbara after clearing $\mathrm{HH}$ and $\mathrm{HL}$ in $\mathbf{5 / 3}$ Filter

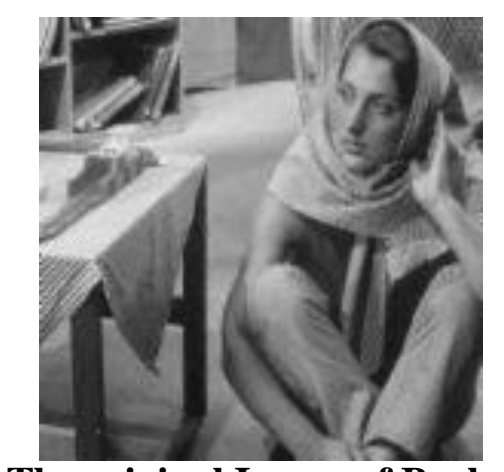

The original Image of Barbra

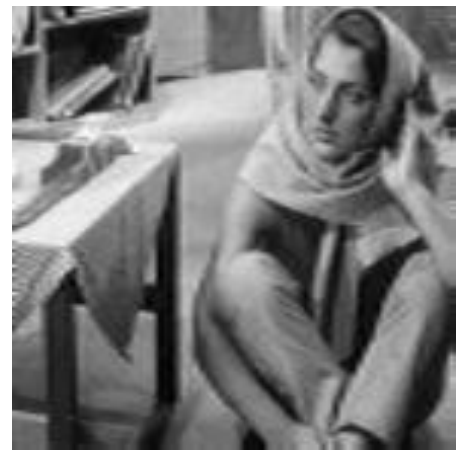

Reconstructed Image of Barbara after clearing $\mathrm{HH}$ and $\mathrm{HL}$ in 9/7 Filter 


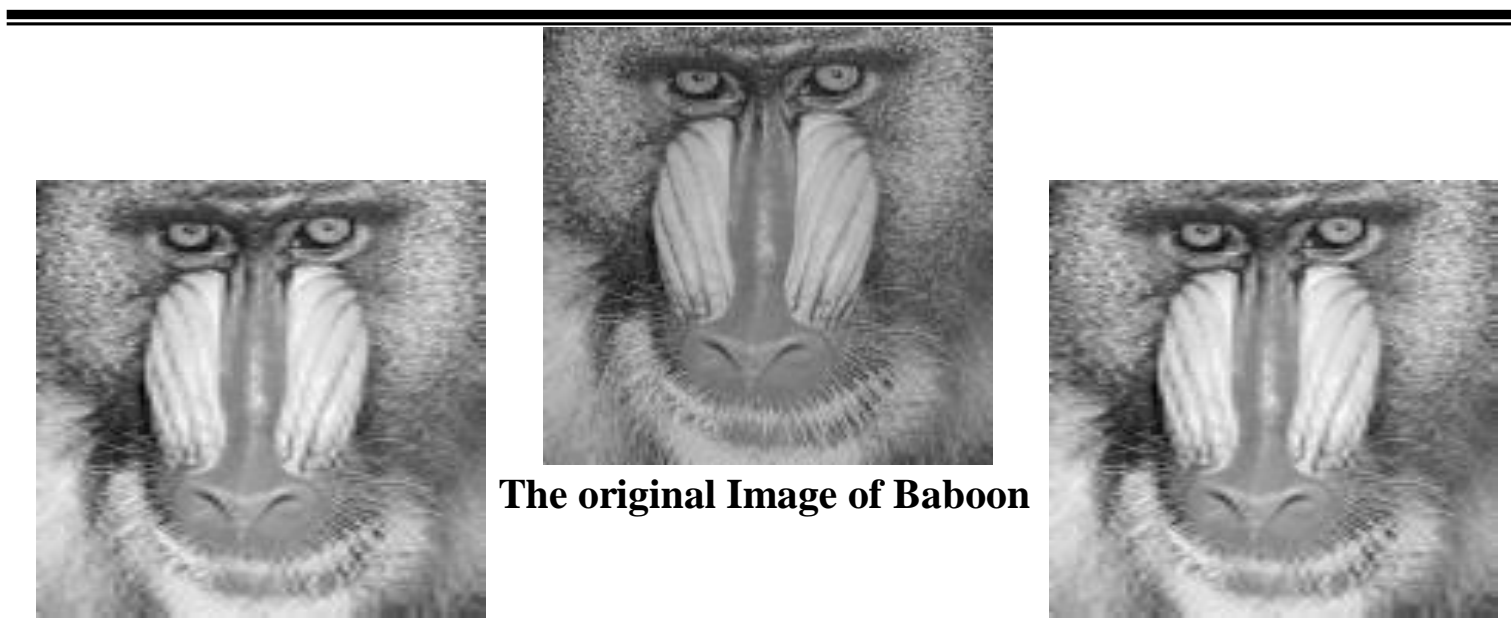

Reconstructed Image of Baboon after clearing $\mathrm{HH}$ and $\mathrm{HL}$ in 5/3 Filter

Reconstructed Image of Baboon after clearing $\mathrm{HH}$ and HL in 9/7 Filter

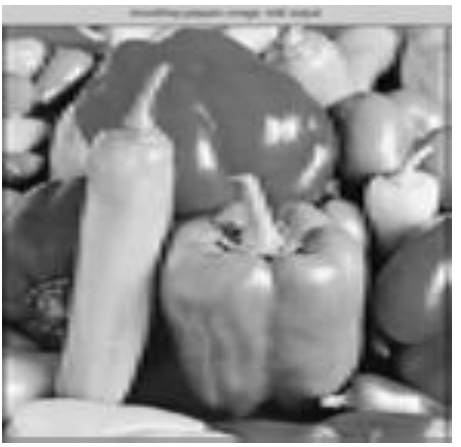

Reconstructed Image of Paper after clearing HH and HL in 5/3 Filter

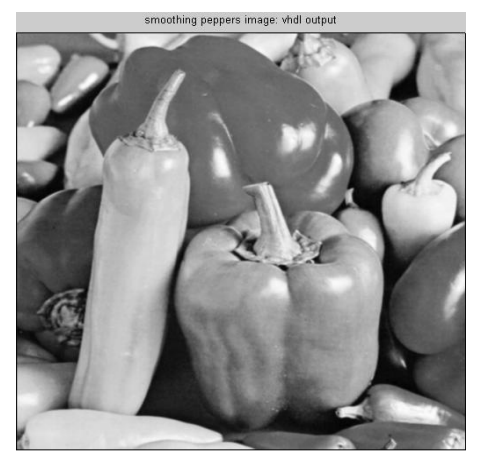

The original Image of Papers

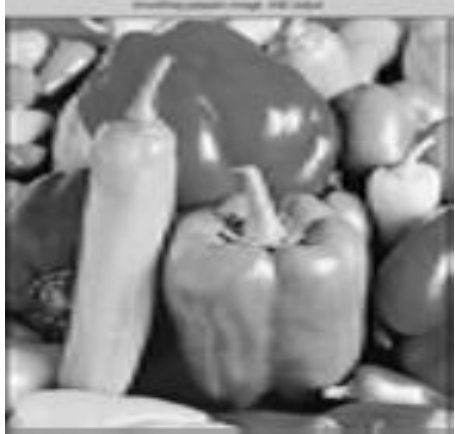

Reconstructed Image of Paper after clearing HH and HL in 9/7 Filter

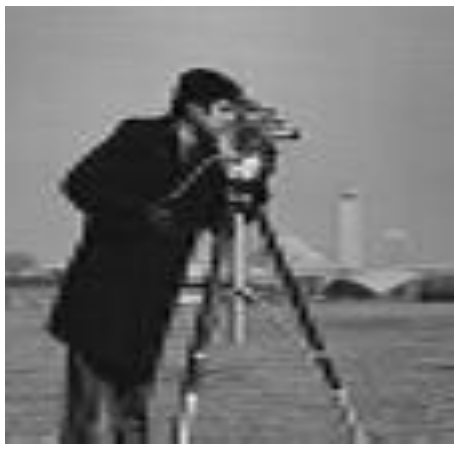

Reconstructed Image of Camera man after clearing $\mathrm{HH}$ and $\mathrm{HL}$ in $5 / 3$ Filter

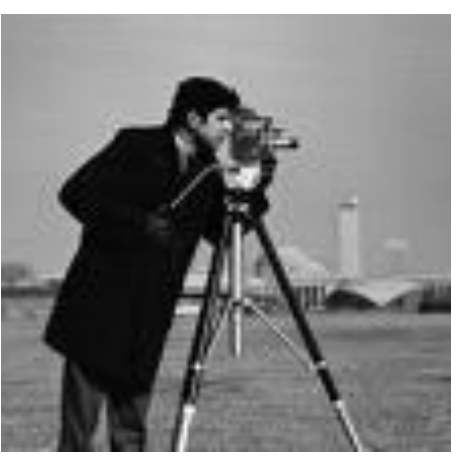

The original Image of Camera man

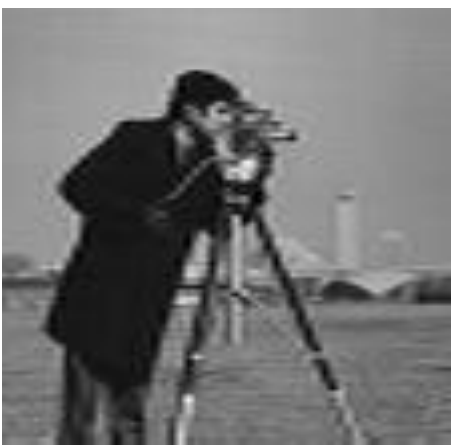

Reconstructed Image of Camera man after clearing $\mathrm{HH}$ and $\mathrm{HL}$ in 9/7 Filter

Fig.19 The reconstruction images of 5/3 and 9/7 filters. 
Table.3 The SNR results from clearing HH, HL and LH sub-band images.

\begin{tabular}{|c|c|c|}
\hline $\begin{array}{c}\text { Images } \\
145 X 145\end{array}$ & $\begin{array}{c}\text { SNR(dB) by } \\
\text { using 5/3 filter }\end{array}$ & $\begin{array}{c}\text { SNR(dB) by } \\
\text { using 9/7 filter }\end{array}$ \\
\hline Barbara & 49 & 51.35 \\
\hline Baboon & 51.80 & 52.68 \\
\hline papers & 37.43 & 36.79 \\
\hline Camera man & 34.46 & 35.55 \\
\hline
\end{tabular}

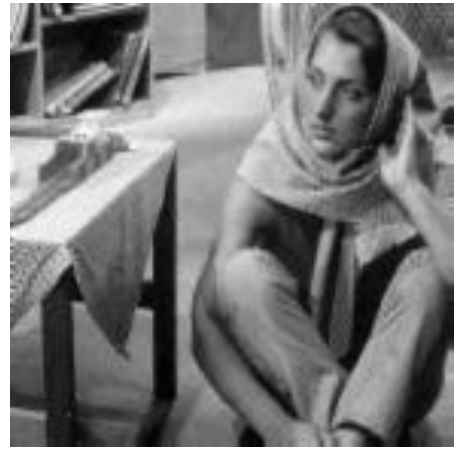

Reconstructed Image of Barbara after clearing $\mathrm{HH}, \mathrm{HL}$ and $\mathrm{LH}$ in $\mathbf{5 / 3}$ Filter

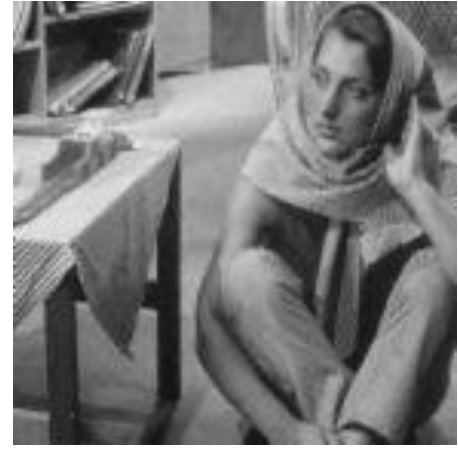

The original Image of Barbara

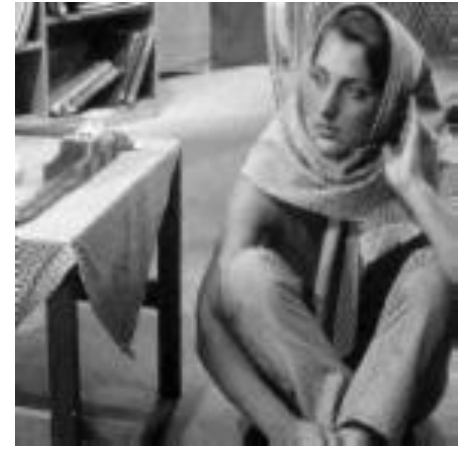

Reconstructed Image of Barbara after clearing HH, HL and LH in 9/7 Filter

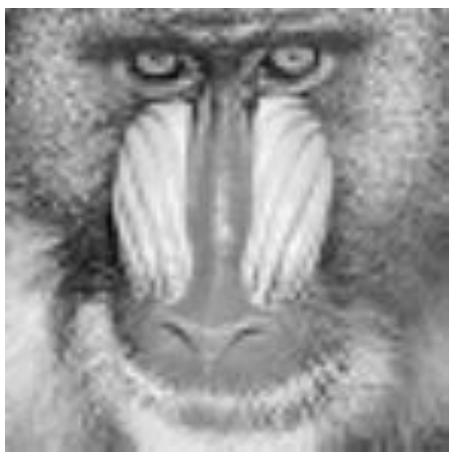

Reconstructed Image of Baboon after clearing HH, HL and LH in 5/3 Filter

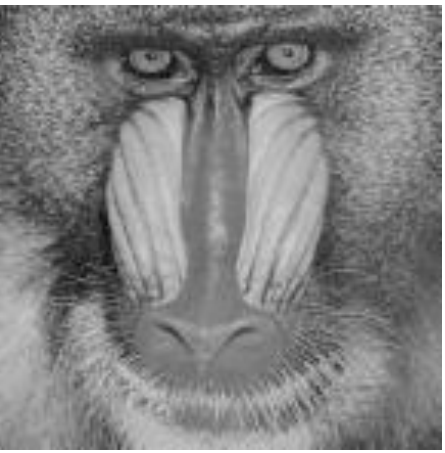

The original Image of Baboon

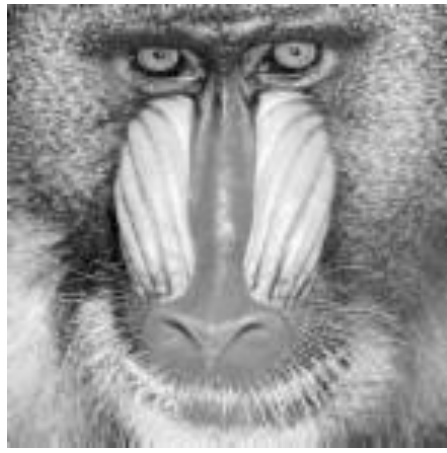

Reconstructed Image of Baboon after clearing $\mathrm{HH}$, HL and $\mathrm{LH}$ in 9/7 Filter 


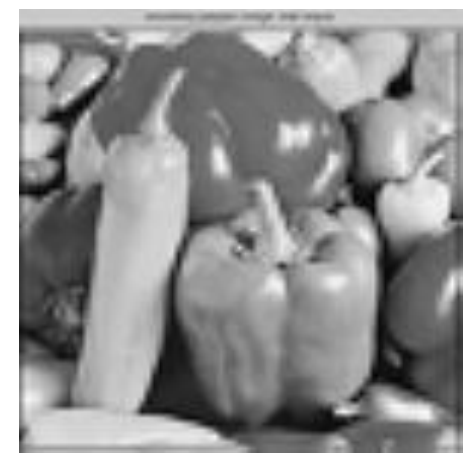

Reconstructed Image of Papers after clearing $\mathrm{HH}, \mathrm{HL}$ and $\mathrm{LH}$ in $\mathbf{5 / 3}$ Filter

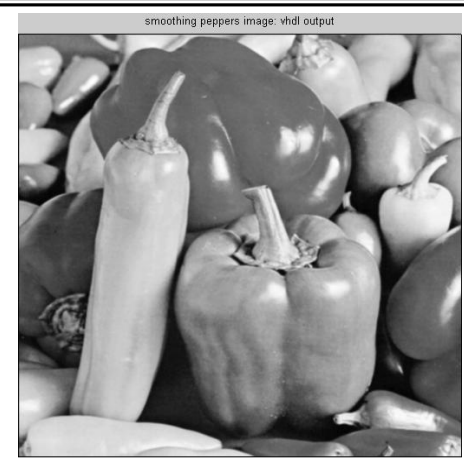

The original Image of Papers

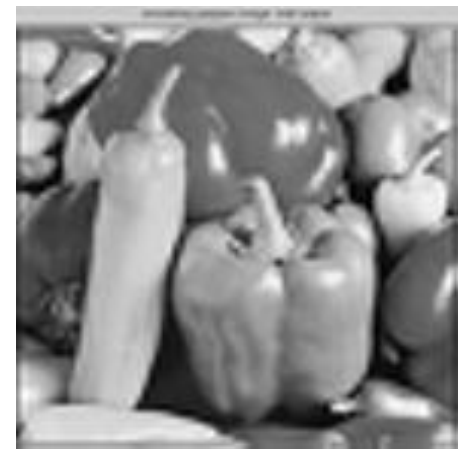

Reconstructed Image of Papers after clearing HH, HL and LH in 9/7 Filter

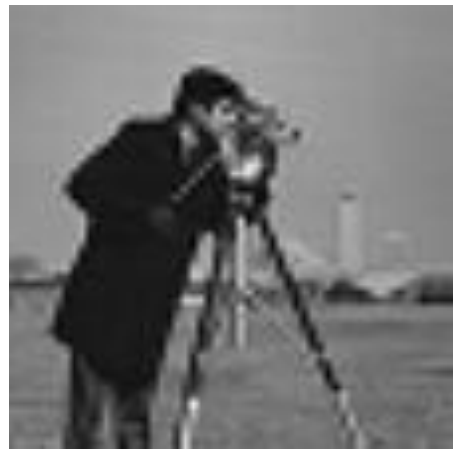

Reconstructed Image of Camera man after clearing $\mathrm{HH}, \mathrm{HL}$ and LH in 5/3 Filter

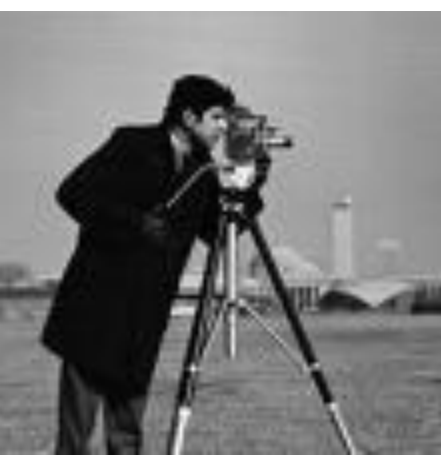

The original Image of Camera man

Fig. 20 The reconstruction images of $5 / 3$ and $9 / 7$ filters.

\section{Conclusion}

The clearing of HH sub-band image produce the maximum SNR compare with the SNR that generate from clearing $\mathrm{HH}$ and HL sub-band images and the SNR which generated from clearing HH, HL and LH sub-band images. The clearing process which is performed into LH and HL sub-band images, deleted some of the low frequency pixels which represented important information, But the clearing process decreases the size of pixels that needed to reconstruct the image, i.e. the number of pixels is needed to reconstructed the image is $\mathrm{N}^{2}$ but when the clearing process is used the number of pixels is 


\section{Dhafer Fakhery Hasan Al-layla}

$3 \mathrm{~N}^{2} / 4$ if the clearing process is applied into $\mathrm{HH}$ sub-band image and $\mathrm{N}^{2} / 2$ if the clearing process is applied into $\mathrm{HH}$ and $\mathrm{HL}$ sub-bands images and $\mathrm{N}^{2} / 4$ if the clearing process is applied into $\mathrm{HH}, \mathrm{HL}$ and $\mathrm{LH}$ sub-band images). So it must be tradeoff between the cleaning information of the image and the size of sub-bands images which used to reconstructed the image to reduce the external memory.

\section{References}

1) I. Daubechies and W. Sweldens, "Factoring wavelet transforms into lifting schemes," J. Fourier Anal. Appl., vol. 4, pp. 247-269, 1998.

2) Chung- Jr Lain, Kunan- Fu Chen, Hong- Hu Chen and Liang- Gee Chen"Lifting Based Discrete Wavelet Transform architecture for JPEG 2000 "IEEE International Symposium on Circuits and Systems, ISCAS 2001,VOL. 2, Page(s): 445 - 448, 2001.E- mail: \{cjlian, lgchen\} @ video.ee.ntu.edu.tw.

3) O. Fatemi and S. Bolouki," Pipline, Memory - efficient and programmable architecture for 2D discrete wavelet transform using lifting scheme "IEE Proc. -circuits Devices sust., VOL. 152, NO. 6, December 2005.

4) K-C.B. Tan and T. Arslan "Shift - Accumulator ALU centric JPEG2000 5/3 lifting scheme based on discrete wavelet transform architecture" 0-7803-7761-3/03/\$17.00 @ 2003 IEEE.

5) Po-Cheng $\mathrm{Wu}$ and Liang- Gee Chen "An efficient architecture for two-dimensional discrete wavelet transform" IEEE Trans. Circuits and Systems for video technology, vol. 11, NO.4, April 2001.

6) T. Acharya, "A high speed systolic architecture for discrete wavelet transforms," in Proc. IEEE Global Telecomm. Conf., vol. 2, 1997, pp.669-673.

7) A. Grzeszczak, M. K. Mandal, S. Panchanathan, and T. Yeap, "VLSI implementation of discrete wavelet transform," IEEE Trans. VLSI Syst., vol. 4, pp. 421-433, June 1996.

8) Juyoung Kim and Taegeun Park" High Performance VLSI Architecture of 2D Discrete Wavelet Transform with Scalable Lattice Structure" World Academy of Science, Engineering and Technology 542009. 
9) Pei - Yin Chen "VLSI implementation for one - Dimensional Multilevel lifting-Based Wavelet Transform "IEEE Transaction on computers, VOL. 53, NO. 4, April 2004.

10) Chao - Tusng Hung, Po- Chih Tesng and Liang-Gee "Anlysis and VLSI archityecture for 1-D and 2-D Discrete Wavelet Transform "IEEE Transaction on Signal Processing, VOL. 53, NO.4, April 2005.

11) Kishore Andra, Chaitali Chakrabarti,and Tinku Acharya, "A VLSI Architecture for Lifting-Based Forward and Inverse Wavelet Transform" IEEE TRANSACTIONS ON SIGNAL PROCESSING, VOL. 50, NO. 4, APRIL 2002.

12) Tinku Acharya, Ping-Sing-Tsai" JPEG2000 Standard for Image Compression" Awiley-interscience publication, ISBN:0-471-48422-9 (cloth) 2005.

13) K. Masselos, Y. Andreopoulos and T. Stouraitis "Performance comparison of two -dimensional discrete wavelet transform computayion schedules on a VLIW digital signal processor" IEE Proc.-Vis. Image Pocess., VOL.153, NO. 2, April 2006.

14) R. Madhusudhanan and A. Arun, "A Novel Architecture Based DWT with Folded and Pipelined Schemes for Infrasound Signal Classification" International journal of mc square scientific research, Vol. 1, June 2009. 